\title{
H. Worth
}

\section{Disease Management Programme für Asthma und COPD: Fortschritt oder Rückschritt?}

\author{
Disease Management Program for Asthma and COPD - \\ a Step Forward or Backward?
}

Im August 2001 stellte der Sachverständigenrat für die konzertierte Aktion im Gesundheitswesen fest, dass die Versorgung von Patienten mit Asthma und COPD verbesserungsbedürftig sei. Für das Asthma Management wurden ein zu geringer Anteil an präventiven Maßnahmen und Qualitätsmängel in der Allergiediagnostik bemängelt, ferner eine unzureichende, nicht flächendeckende Versorgung mit strukturierten Patientenschulungen. Inhalative Kortikosteroide, die die Basistherapie des Asthmas darstellen, werden nach Auffassung des Sachverständigenrates zu selten, kurzwirksame Beta-2-Agonisten, Xanthinderivate und Mukopharmaka zu häufig eingesetzt. Bezüglich des COPD-Managements wurden parallel hierzu ein unzureichender Einsatz der Tabakentwöhnung kritisiert, ferner eine im Verlauf der Erkrankung zu spät einsetzende und nicht leitliniengerechte Diagnostik. Es bestünde ein erheblicher Interventionsbedarf zur Umsetzung leitliniengerechter Therapieempfehlungen und ein Entwicklungsbedarf bezüglich strukturierter Patientenschulungen.

Im Rahmen der Umsetzung des Gesetzes zur Reform des Risikostrukturausgleichs in der gesetzlichen Krankenversicherung wurde beschlossen, zur Verbesserung der Versorgung von Patienten mit chronisch obstruktiven Atemwegserkrankungen, die Durchführung strukturierter Behandlungsprogramme für diese Krankheiten im Risikostrukturausgleich finanziell zu fördern. Inzwischen liegt ein Referentenentwurf vor, der die Mindestanforderungen für die Zulassung von Disease Management Programme für das Asthma bronchiale und die COPD im Rahmen einer Rechtsverordnung beschreibt. Außerdem wird in den Entwurf die für die Durchführung strukturierter Behandlungsprogramme für Patienten mit Asthma bronchiale bzw. COPD erforderliche
Dokumentation festgelegt. Der Referentenentwurf hat gute Chancen, im Jahre 2005 verwirklicht zu werden.

In diesem Entwurf zum Disease Management Programm (DMP) Asthma/COPD soll die Behandlung der Patienten auf der Basis evidenzbasierter Leitlinien unter Berücksichtigung der jeweiligen Versorgungssektoren erfolgen.

Beide Krankheitsbilder sowie die Kriterien für den Einschluss in das DMP wurde sorgfältig definiert. Die notwendige Diagnostik des Asthma bronchiale und der COPD auf der Basis von Anamnese, Symptomatik, körperlicher Untersuchung, Kenngrößen der Lungenfunktion und zusätzlicher Verfahren wurden charakterisiert.

Zur Erreichung der Therapieziele, der Vermeidung von akuten und chronischen krankheitsbedingten Beeinträchtigungen und der Reduktion der krankheitsbedingten Letalität, wurden allgemeine Maßnahmen unter Einschluss der Infektionsprophylaxe, die Aufgabe des Tabakrauchens, insbesondere bei Patienten mit COPD, nicht medikamentöse Maßnahmen unter Berücksichtigung der strukturierten Patientenschulung und auch des körperlichen Trainings sowie die notwendige medikamentöse Therapie charakterisiert. Als Indikatoren zur Beurteilung der Versorgungsqualität wurden unter anderen Kenngrößen der Anteil der Patienten mit Asthma/COPD eines Arztes angeführt, der einer stationären und/oder ambulanten Notfallbehandlung in einem bestimmten Zeitraum bedarf, ferner der Anteil, bei denen im Dokumentationsraum Empfehlungen zum Verzicht des Tabakrauchens bzw. zur Nutzung eines körperlichen Trainingsprogramms 
gegeben wurden, schließlich auch der Anteil der Patienten, der an einer strukturierten Patientenschulung teilnimmt.

Der Nutzen des DMP für Patienten mit Asthma und COPD dürfte die bessere Erfassung beider Patientengruppen durch eine adäquate Diagnostik sein. Vorteil für den betroffenen Patienten sind ferner eine leitliniengerechte medikamentöse Therapie und eine stärkere Nutzung nicht medikamentöser Therapieverfahren wie der strukturierten Patientenschulung für Asthmatiker und COPD-Patienten, ferner die Raucherentwöhnung und das körperliche Training, insbesondere bei COPD-Patienten. Potenzielle Risiken betreffen den höheren initialen Kostenaufwand bei der Erfassung und Erstdiagnostik der Patienten mit obstruktiven Atemwegserkrankungen, die möglicherweise Änderungswünsche bezüglich der Ausführungen des DMP's bei den Kostenträgern induzieren.

Der mit der Verwirklichung des DMP Asthma/COPD verbundene höhere Dokumentationsaufwand, der trotz aller Bemühungen, die Zahl der zu dokumentierenden Parameter möglichst gering zu halten, entstehen wird, kann zu einem Verdruss der behandelnden Ärzte beitragen und dazu Anlass geben, Patienten dem DMP fernzuhalten. Weitere Risiken betreffen die Verzögerung des Eingangs innovativer Therapieverfahren in das DMP, da diese nicht immer zeitnah einem Prüfverfahren zur Aufnahme in das DMP unterliegen. Auch eine unzureichende Umsetzung der Qualitätssicherung innerhalb des DMP's kann die Effizienz des DMP Asthma/COPD belasten. Insgesamt bietet das DMP Asthma/COPD jedoch die große Chance, Patienten mit Asthma bronchiale und COPD häufiger als bisher durch eine adäquate Diagnostik zu erkennen und sie effektiver als bisher zu behandeln.

Im vorliegenden Heft berichten Petro und Mitarb. [1] über den Einsatz eines Disease Management Programms für Asthmatiker, der in einer randomisierten und kontrollierten Studie geprüft wurde. Speziell geschulte, nicht ärztliche Case-Manager betreuten neben Hausarzt und Facharzt die in die Studie eingeschlossenen Patienten, führten Patientenschulungen zu asthmabezogenen Themen durch und überwachten per Modem übertragener krankheitsbezogener Daten den Krankheitsverlauf der Patienten.
Die Auswertung erfolgte mit Hilfe definierter Algorithmen eines speziell entwickelten Programms. Auf fehlende oder unplausible Daten wurde umgehend reagiert. Im Vergleich zu einer nicht über Case-Manager behandelten Kontrollgruppe zeigten sich bessere Werte von Kenngröße der Lebensqualität, eine diskret bessere Lungenfunktion, niedrigere direkte, aber höhere indirekte Kosten. Die Studie wurde bei älteren Erwachsenen mit mittelschwerem und schwerem Asthma durchgeführt, so dass ihre Ergebnisse sicherlich keine volle Gültigkeit für Kinder, Jugendliche und junge Erwachsene mit Asthma bronchiale haben dürften. Die positiven Effekte auf die Besserung der Symptomatik dürfte durch die mittels intensiverer Überwachung erreichten besseren Kontrolle der Erkrankung und des höheren Einsatzes inhalativer Kortikosteroide zu erklären sein. Ob das Programm kosteneffektiv ist, kann nicht eindeutig beantwortet werden, da die Kosten für den Einsatz der Case-Manager sowie für die gegenüber PeakFlow-Metern aufwändigeren Asthma-Monitore in eine KostenNutzen-Analyse nicht einbezogen wurden. Die Studie zeigt, dass durch den Einsatz von Case-Managern und durch speziell zur Überwachung des Krankheitsverlaufs und von Therapieentscheidungen entwickelten Programme wahrscheinlich eine bessere Kontrolle der Erkrankung möglich ist.

Ob sich der Einsatz von nichtärztlichen Case-Managern als zusätzlichen Betreuern von Asthmatikern neben Hausarzt und Facharzt im Sinne einer Optimierung des Asthmamanagements bewährt, kann erst durch weitere Untersuchung an größeren Patientenkollektiven über längere Zeiträume geklärt werden. Bereits heute ist hingegen gesichert, dass eine effiziente, strukturierte Patientenschulung bei erwachsenen Asthmatikern zu einer Besserung der Lebensqualität, einer Reduktion der Morbidität und gleichzeitiger Reduktion der Kosten für die Behandlung erwachsener Asthmatiker führt.

\section{Literatur}

${ }^{1}$ Petro W, Schulenburg J v.d., Greiner W et al. Effizienz eines Disease Management Programmes bei Asthma. Pneumologie 2005; 59: $101-107$ 